Journal of

Accident and

Emergency

Medicine 1994

11, 183-185

\title{
Review of the composition and use of trauma teams within the Trent Region
}

\author{
D.A. HIGHLEY \\ Royal Hallamshire Hospital, Glossop Road, Sheffield
}

\section{SUMMARY}

The aim of the study was to find the existence, composition and working policies of trauma teams within the Trent Region. Data were obtained by postal survey. Despite many reports indicating a need for a unified multidisciplinary approach to trauma management, this study found that this approach was absent in many hospitals.

Key words: trauma, trauma team

\section{INTRODUCTION}

The current plan for trauma care in this country is unclear. Regional or sub-regional trauma centres in line with developments in North America have been proposed. Stoke-on-Trent is the only centre that has been established so far and is a pilot site. Until such time as trauma centres are introduced into the UK, district general hospitals will continue to manage multiply injured patients using their current staff and resources. It would seem sensible to develop a trauma team concept in these hospitals to supplement the junior staff available within accident and emergency (A\&E) departments especially outside normal hours.

The concept of dedicated groups within hospitals to manage life threatening conditions is not a new one; most acute hospitals have cardiac arrest teams which operate throughout the hospital. These teams often use management algorithms such as those proposed by the United Kingdom Resuscitation

Correspondence:

D.A. Highley, Anaesthetic Registrar, Department of Anaesthetics, Aberdeen Royal Infirmary, Foresterhill Road, Aberdeen AB9 2ZB, UK
Council. Trauma teams that are organized in a similar way are found both outside and within the UK. ${ }^{1,2}$ The advantage of having experienced additional help that arrives en masse, rather than in a piecemeal fashion, may reduce the hiatus in treatment that occurs while treatment plans are organized or while staff arrive from home.

The aim of this study was to survey the use of trauma teams within Trent Region, their component members and how they were deployed. Trent Region was chosen as it has a combination of urban and rural hospitals and four teaching hospital centres.

\section{METHODS}

Fifteen hospitals were identified in the region as having full-time general A\&E departments and College of Anaesthetists tutors. Each hospital was sent a questionnaire. Those centres not replying within 2 months were sent a second questionnaire producing a $100 \%$ response rate.

\section{RESULTS}

All 15 hospitals responded, of which five had established trauma teams. The teams consisted of anaesthetists, general surgeons and orthopaedic surgeons in addition to the staff available in the A\&E department. Their experience level varied between senior house officer and consultant. One of the hospitals had the A\&E registrar as designated team leader, with two other hospitals indicating that senior A\&E staff would be summoned, their role presumably being that of team leader. None of the four teaching hospitals in the region had trauma teams.

In all hospitals with teams, the initiator of the callout was a member of the A\&E staff. In one department the nurse in charge of the A\&E was authorized to call the team without reference to medical staff, following triage provided that the patient fulfilled certain predetermined criteria. $^{3}$ Other hospitals indicated that the assessment and decision to call the team was a medical staff decision and therefore there was no specified role for triage by nursing staff. This may change as the role of A\&E nurses evolves.

Management protocols were used in seven hospitals and these were combined with structured 
Table 1. Component members of trauma teams. $X$ indicates those staff providing cover during a 24-h period (not all staff were available for the full 24 hours)

\begin{tabular}{|c|c|c|c|c|c|c|c|c|c|}
\hline \multirow[b]{2}{*}{ Hosp No } & \multicolumn{3}{|c|}{ Anaesthetic. } & \multicolumn{3}{|c|}{ General Surgery } & \multicolumn{3}{|c|}{ Orthopaedics } \\
\hline & $\mathrm{SHO}$ & Reg & SR/Cons & $\mathrm{SHO}$ & Reg & SR/Cons & $\mathrm{SHO}$ & Reg & SR/Cons \\
\hline 1 & $x$ & $x$ & $x$ & & $x$ & $x$ & & $x$ & $x$ \\
\hline 2 & $x$ & & $x$ & $x$ & & $x$ & $x$ & & $x$ \\
\hline 3 & & $x$ & & & $x$ & & & $x$ & \\
\hline 4 & $x$ & $x$ & & $x$ & $x$ & & $x$ & $x$ & \\
\hline 5 & & $x$ & $x$ & & $x$ & $x$ & & $x$ & $x$ \\
\hline
\end{tabular}

patient assessment forms in three cases. Only one hospital had a trauma team, management protocols and a structured patient assessment form. Review of the activity, response and outcome of cases where the trauma teams were involved were reviewed in one hospital but no figures were requested. The remaining trauma teams had only recently been instituted and no data were available although a regular audit of their activities was to be undertaken.

Training of team members was undertaken in three hospitals. In two it was based on Advanced Trauma Life Support (ATLS) guidelines and in the other hospital it was based on a locally derived scheme. Of the 44 consultant anaesthetists in the five hospitals with trauma teams five were ATLS trained. With the rapid change in junior staff and the priority given to consultants in ATLS training it was decided that the training and permanent resources would give a better indication of the ability of the team members and for this reason only the ATLS training of the Consultants was considered.

\section{DISCUSSION}

Multiple injuries are a relatively rare but important occurrence in Great Britain, accounting for $0.5 \%$ of A\&E patients. ${ }^{4}$ Most doctors therefore have limited experience in the management of major trauma and its associated problems.

Treatment protocols may stifle the management skills of medical staff, but in a simple task such as diagnosis and management of hypotension, protocols have been shown to improve patient outcome, with a decrease in resuscitation time and a decrease in the incidence of related complications. ${ }^{5}$ Management protocols were used in only $46 \%$ of hospitals despite their value. The Advanced Trauma Life Support Course (ATLS) treatment protocols simplify patient management and focuses the attention of the medical staff on life threatening problems and away from peripheral injuries. ATLS provides a structured format to encourage full patient assessment treatment and review. ${ }^{6}$ With more medical staff undertaking training courses it provides a common core for all medical staff involved in trauma patient care regardless of their place of employment.

The use of structured patient assessment forms has advantages. In addition to simply organizing the documentation of injuries they also act as a diagnostic aide memoir to improve the accuracy of diagnoses made by trainees. ${ }^{7}$ Only $20 \%$ of hospitals in this survey had taken this step which would be a simple way to improve patient outcome.

The decision to summon the trauma team was made by the medical staff in the A\&E department in all but one hospital, where the nurse in charge of the department was authorized to call the team if certain predetermined criteria were fulfilled. Review of this system showed that all of the patients with significant trauma fulfilled one or more of the criteria for the trauma team to be called. ${ }^{3}$ The expansion of the role of senior nurses in the A\&E department to encompass triage of all patients means that with appropriate guidance high-risk patients can be identified by physiological scoring and appropriate action taken soon after a patients arrival in the department. The use of trauma scoring by nursing staff during triage or by medical staff to highlight physiologically abnormal patients has improved patient outcome ${ }^{8}$ and provides a means of identifying ill patients in whom there may be no obvious physical cause.

Recent reviews of trauma management have commented on the poor standard of trauma care in this country ${ }^{9-11}$ and the need to improve care by the use of trauma centres following the improvement in care found in the United States after their introduction. ${ }^{12}$ Until this time the existing trauma services will need to improve their standard of care with the resources available in each of their hos- 
pitals. Trauma teams and structured, standardized documentation could decrease delays in treatment and improve diagnostic accuracy.

The choice of Trent region as a sample for this study may not be representative of the whole country, but the problems in providing an efficient trauma service are universal. Although the proportion of hospitals with trauma teams, treatment protocols, triage and structured assessment forms may vary the combination of these together in one unit is absent, with only one hospital in this study combining all components in a patient management package.

\section{ACKNOWLEDGEMENTS}

I would like to thank the Royal College of Anaesthetists Tutors for completing the forms, Dr P. Mathews, Dr T. Shaw and Mr T. Beattie for their advice and support during the planning, execution and completion of this study.

\section{REFERENCES}

1. Spencer J.D. (1985) Why do our hospitals not make more use of the concept of trauma teams? British Medical Journal 290, 136.

2. Deane S.A., Gaudry P.L., Pearson I. et al. (1983) Implementation of a trauma team. Australia and New Zealand Journal of Surgery 59, 373.

3. Over D. \& Finch M. (1991) The development of new documentation for use in cases of major trauma. Injury 22 (2), 139.

4. Royal College of Surgeons of England (1988) Report of the working party on the management of patients with major injuries. Royal College of Surgeons of England, London.

5. Hopkins J.A., Shoemaker W.C., Chang P.C., et al. (1983) Clinical trial of an emergency resuscitation algorithm. Critical Care Medicine 11, 621.

6. Advanced Trauma Life Support Course, Student Manual (1989) American College of Surgeons, Bethesda.

7. Adams I.D., Chan M., Clifford P.C., et al. (1986) Computer aided diagnosis of acute abdominal pain: a multi centre study. British Medical Journal. 293, 800.

8. Rutherford W.H. (1990) Reception of severely injured patients in hospital. Injury 21 (6), 344.

9. Anderson I.D., Woodford M., De Dombal T., et al. (1988) A retrospective study of 1000 deaths from trauma in England and Wales. British Medical Journal 296, 1305.

10. Buck N., Devlin H.B. \& Lunn J.N. (1987) Report of a confidential enquiry into perioperative deaths. Nuffield Provincial Hospitals Trust, Kings Fund Centre, London.

11. Yates D.W., Woodford M. \& Hollis S. (1992) Preliminary analysis of the care of injured patients in 33 British hospitals: First report of the United Kingdom Major Trauma outcome study. British Medical Journal 305, 737.

12. West J.G., Cales R.H. \& Gazzaniga A.B. (1983) Impact of regionalisation. The Orange County Experience. Archives of Surgery 118, 774. 